XXVIII.

Aus der medicinischen Universitätspoliklinik Bern

(Director: Prof. Sahli; Assistenzarzt: Priv.-Doc. Dr. F. Seiler).

\title{
Beitrag zur Kenntniss der Wirkung von Eisen und Arsenik als Mittel gegen die Chlorose.
}

\author{
Von
}

\begin{abstract}
Anna Zwetkoff aus St. Petersburg.
\end{abstract}
Die Behandlung der Chlorose mit Eisen und Arsenik ist zur allgemein gültigen Regel geworden, so dass in den medicinischen Lehrbüchern neben diätetisch-hygienisehen Vorsehriften stets die Anwendung von Eisen und Arsenik empfohlen wird. In der neuen Jiteratur dreht sich die Frage auch nicht mehr darum, ob das Eisen wirksam sei oder nicht, sondern es wird hauptsäehlich die Frage discutirt, welehes von den vielen Eisenpräparaten das wirksamste sei. Am meisten Beliebtheit besitzen auch heute noch die längst bekannten Blaud'schen Pillen, die auch in den verbreiteten Lehrbüchern von Strümpell, Eichhorst, v. Mering, Grawitz, Niemeyer, Laache etc. besonders empfohlen werden. Auch auf der Sahli'schen Klinik wird das Eisen, wenn irgend möglich, in Form von Blaud'schen Pillen verabfolgt und damit in der grossen Mehrzahl der Fälle innerhalb relativ kurzer Zeit die Heilung der Chlorose erzielt.

Ich konnte aus dem Material der Klinik von Prof. Sahli 33 Fälle von Chlorose zusammenstellen, beidenen der Hämoglobingehalt des Blutes bei einer Behandlung mit Blaud'schen Pillen von durehsehnittlich 41 Tagen Daver in prompter Weise anstieg, so dass der Hämoglobinwerth, der im Anfang der Behandlung im Mittel 42 betrug, am Ende der Behandlung nach ca. 6 Wochen im Mittel 85 erreichte. Dabei macht Prof. Sahli stets darauf aufmerksam, dass verhältnissmässig grosse Dosen Blaudseher Pillen zur Erreichung einer prompten Wirkung verordnet werden müssen.

Ueber die unendlich zahlreichen organischen und unorganischen Eisenpräparate, welche als Ersatz für Blaud'sche Pillen zur Verwendung kommen, besteht eine nicht zu bewältigende Literatur. Bei den Untersuchungen dieser Präparate auf ihre Wirksamkeit wird hauptsächlich darauf Rücksicht genommen, ob dieselben von den Patienten gut ertragen werden und ob damit cin Ansteigen des Hämoglobinwerthes und eventl. der Erythrocytenzahl erreicht werden kann. 
Es fällt einem aufmerksamen Leser dabei auf, dass über die Resultate der Behandlung von Bloichsüchtigen mit den verschiedenen Präparaten fast ausnabmslos in ricmlich unbestimmter Weise geurteilt wird, da man meistens Angaben findet, dass \%. B. „in wenigen Wochen allgemeine Besserung eintrat", oder dass dieses oder jenes Präparat "das wirksamste sei". Ls fällt auf, dass in der grossen Literatur über Chlorosebehandlung mit Eisenpräparaten nur ganz ausnahmswcise regelmässige, exakte Untersuchungen über die Fortschritte der Hämoglobinbildung and die successive Zunahme der livythrocyten in den. Fallen, wo dieselben vermindert waren, angestellt worden sind. Einzig derartige Untersuchungen würden feststellen lassen, ob ein Untersehied in der Wirksamkeit der verschiedenen lisenpräparate wirklich besteht oder nicht.

Einigermaassen genauere Angaben über den Heilungsverlauf der Chlorosen finden sich bei folgenden Autoren:

Biernatzki (1) hält die grossen Dosen von Eisen für besonders wirksam, denn aus Vergleichen, die or mit den Resultaten der Eisenwasserbohandlung zog, erhielt er folgendes Ergebriss: bei der Behandlung mit Fisenpillen nahm die Kahl der rothen Blutkörperehen durehsetmittlich um 58790, bei Behandlung mit Eisenwässern um 40410 pro Behandlungstag zu.

Nach Neuss ist das Ferrum phosphoricum cum Natrio citrico bei der Chlorosebehandlung völlig geeignet. Letzteres Präparat wurde auch von Graeber in einem Fall versucht. Bei einer täglichen Injection von 2 ecm einer lüsung von 1:30, später 1:6 steigt der Hämoglobingehalt in 54 Tagen von 5,6 auf 10,9 pCt.

Riemer u. Frisch rühmen nach den im Wiener Allgcmeinkrankenhause unter Drasche gemachten Erfahrungen das Carniferrin bei Anämie und Chlorose, indem es zu $0,34 \times$ tïgl. bei gewöhnlicher Spitalkost, auch ohne Bettruhe unter Schwinden der subjectiven Beschwerden innerhalb 1 Woche bei Chlorose das Hämoglobiri um 3 und später um 10 pCt. steigerte.

Von zahlreichen anderen Autoren wird meistens nur angegeben, dass boi Anwendung eines gewisson Eisenpraparates in 3-4-6 Woehen in der Regel Heilung erielt worden sei.

Was die Behandlung der Chlorose mit Arsenik anbetrifft, so wird seine Wirkung im allgemeinen viel geringer taxirt als die Wirkung des lisens, nur hier und da fiuden sich Angaben äber günstige Resultate.

v. Noorden (2) sigt vom Mrsen, dass inm cin amregender Binfluss auf das Knochenmark zukommt.

Strümpell (3) äussert sich, dass das Arsenik als ein Mittel zu nennen ist, das zuweilen einen eigenthümlich günstigen Einfluss auf die Gesammtconstitution ausübt und daher in schwereren Fällen von Chlorose manchmal Anwendung verdient.

Widal u. Hirtz (4) sprechen sich günstig ans über die Verwendbarkeit des kakorjlsauren Natriums bei Chlorose. Bei Tagesgaben von 0,05-0,1 subcutan konnte Widal Zunahme der Erythrocyten constatiren, die eine \%eit lang nach dem Aussetzen des Mittels persistirte, jedoch 
nicht über die Normalzahl hinausging. Die Hämoglobinmenge wird auch vermehrt, jedoch nicht proportional der Zahl der Erythrocyten.

Für Beeinflussung der Chlorose verwandte Brenner (5) ein natürliches Arsenwasser, die Maxquelle in Leviko. Mit einer grossen Anzahl von Patienten wurde eine ungefähr $5-6$ wöchentliche Trinkkur durchgeführt, mit kleinen Dosen von $3 \times 15$ ccm beginnend, aufsteigend bis zu $3 \times 90 \mathrm{ccm}$ und ebenso wieder abfallend, was einer Arsengabe von etwa $1 / 4 \mathrm{mg}$ bis zu $1 \frac{1}{2} \mathrm{mg}$ pro Dosis entspricht. Nach Beendigung der Kur war das Blatbild durchgehend ein besseres. Der Hämoglobingehalt war gesteigert, beispielsweise von 32 auf 55 pCt., von 48 auf $70 \mathrm{pCt}$, von 45 auf $68 \mathrm{pCt}$, yon 24 auf $54 p \mathrm{Ct}$.

Die Zahl der Erythrocyten erreichte jetzt in manchen Fallen normale Werthe und hatte sich ofters um eine bis zwei Nillionen vermehrt.

In der "Therapie der Gegenwart", 51. Jahrgang, Heft 2 macht Klemperer (6) Bemerkungen über eine kür»lich veröffentlichte Vorlesung über Chlorose von v. Noorden. Der letetere empfiehlt zur Behandlung der Chlorose Eisen und in solchen Fällen, wo Eisen schlecht vertragen wird, sei es angezeigt, sich des Arsens zu bedienen.

Hilbert u. Lereboullet (7) emplehlen das kakodylsaure Eisenoxyd. Bei innerer Application werden Tagesgaben von $\mathbf{1 5}-25 \mathrm{cg}$ gut vertragen. $K$. und L. haben das Mittel bei Chlorose mit Erfolg benutzt.

Im ganzen sind somit in der Literatur nur spärliche Empfehlungen der reinen Srsenikbeliandlung bei der Chlorose zu finden und im allgemeinen geht die Ansicht dahin, dass die Arseniktherapie erfolglos oder doch nur in geringem Maasse wirksam sei.

Viel baufger als die ausschliessliche Arsenikbehandlung wird die Combination der Eisen- und Arseniktherapie empiohlen.

Nach Neumann spielt das neue Arsenpräparat "Asferryl" eine grosse Rolle bei Chlorosebehandlung. Es enthält $23 \mathrm{pCt}$. Arsen und $18 \mathrm{pCt}$. Eisen, dabei ist es etwa $35 \mathrm{mal}$ ungiftiger wie arsenige Säure.

v. Noorden (8) sagt, dass die Verbindung von Eisen mit Arsen sehr wirksam ist.

Strumpell (9) hat mebrmals Fälle von Chiorose beobachtet, die nach dem Gebrauche von Eisen keine Besserung zeigten, während sie auf Eisen mit kleinen Mengen Arsen $(0,002-0,0043 \mathrm{mal} \mathrm{tägl.)}$ auffallend günstig reagirten.

Gordon (10): Eine Combination des Hisens mit Arsen besitzen wir in den natürhehen eisenarsenhaltigen Wässern, dieselben worden aber nicht immer gut vertragen. Dasselbe gilt auch von den anderen bisher gebräuchlichen Arzneicombinationen von Eisen und Arsen. Es ist deshalb immer noch von Interesse, ein verträgliches lissenarsenpräparat zu kennen. Ein solches Präparat ist das Arsoferrin. Das Präparat hat sich an seiner Klinik gut bewährt. Ein Erfolg war fast immer zu constatiren, da in einer verhäitnissmässig kurzen Zeit der Ernährungszustand der Patienten sich wirklich besserte, das Körpergewicht in die Höhe ging, die Zusammensetzung des Blates normale Werthe erreicht.

Die Behauptung, dass die Behandlung der Chlorose mit Eisen und Arsenik wirksamer sei, als die Eisenbehandlung allein, ist hauptsächlich 
begründet (neben spärlichen exacten Untersuchungen) durch den allgemeinen Eindruck der Praktiker und durch gelegentliche Beobachtung von Fällen, bei denen nach unbefriedigender Eisentherapie der Zusatz von Arsenik eine wesentliche Beschleunigung des Heilungsverlaufes hervorzurufen schien. Ueber die Wirkungsweise dieser beiden Stoffe (Eisen und Arsenik) bei der Behandlung der Chlorose wie überhaupt übrigens auch bei anderen Blutkrankheiten sind die Akten z. Z. noch nicht abgeschlossen. Für das Eisen gilt hauptsächlich die Ansicht, dass es nicht nur einen allfälligen lisenmangel im Organismus ersetat und dadureh eine vormehrte Hämoglobinbildung ermöglicbt, sondern dass es hauptsänlich einen stimulirenden Kinfluss auf die Function derjenigen Knochenmarkzellen ausübt, welche mit der Bildung des Hämoglobins und vielleicht auch der rothen Blutkörperehen betraut sind. Auch Meyer und Gottlieb (11) nehmen eine specifische Wirkung der Eisensalze auf die blutbildenden Organe (Knochenmark) an.

In Uebereinstimmung endlich mit dieser Annahme einer Anregung der blutkörperbildenden Organe dureh Eisensalye stehen auch die histologischen Befunde am Knochenmark. Fr. Müller (12) fand, dass an anämisch gemachten Thieren bei Zufubr von Eisensalzen in der sonst eisenarmen Nabrung das Knochenmark erheblich mehr kernhaltige rothe Blutkörper enthält als das der obno Eisenzufuhr ernährten Controlthiere. Danach werden wir annehmen müssen, dass es sich bei der Eiscnwirkung in der Chlorose um zweierlei Momente handelt: erstlich um die Verwendung des zugeführten lisens als Material zum Aufbau des Hämoglobins und zur Bildung von eisenreichen organischen Reservestoffen in der Leber, Milz und anderen Organen, und zweitens um eine specifisch anregende Wirkung der Eisensalze auf die hämoghobinbildenden Zellen.

Prof. Sahli hat in seiner Vorlesung über die Chlorose die Ansicht geäussert, dass es an Baumaterial den Chlorotischen nicht fehlt. Es könne daher das medicamentös zugeführte Eisen nicht einfach als Baumaterial des Hämoglobins betrachtet werden. Jedenfalls bestehe die Hauptwirkung des Eisens in einer chemischen Reizung zur Bildung von Hämoglobin; dass es sich bei diesem Reizmittel gleichzeitig um das wesentlichste und specifische Baumaterial des Hämoglobins handelt, sei dabei wohl kein Zufall, sondern erkläre sich ohne weiteres durch die Annahme, dass die \%ellen des Organismus wohl gesetzmässig gerade durch diejenigen Stoffe gercizt werden, welche sie verwerthen können. Wenn man Thiere mit Eisen füttert, so findet man, dass das Knochenmark rother wird. Je mehr Fisen dem Knochenmark zur Verfügung gestellt wird, um so mehr hat es Gelegenheit, Hämoglobin zu bilden.

Das Arsenik scheint nach den vorwiegenden Arbeiten und nach den zahlreichen Erfahrungen über ausschliessliche Arsenbehandlung bei der perniciösen Anämie hauptsächlich die Production der rothen Blutkürperchen $z u$ beeinflussen, vielleicht durch Anregung einer intensiveren Production, vielleicht durch Anregung einer Bildung von qualitativ höherstehenden, resistenteren rothen Blutkörperchen.

Es lag nahe diese verschiedenen Fragen der Wirksamkeit von Eisen und Arsenik bei der Chlorosebehandlung an einem grösseren Kranken- 
material genauer zu studiren um, wenn möylich, festzustellen wie sich einerseits der Einfluss dieser Stoffe einzeln und combinirt auf die Bildung des Hämoglobins bei Chlorose verhält und ob andererseits ein Unterschied in der Wirkung auf die Bildung der rothen Blutkörperchen besteht. Man durfte auch hoffen durch derartige genauere Untersuchungen an Kranken eine Bestätigung der vorhandenen experimentellen Befunde zu crzielen.

In der Absicht diese Verbältnisse abzuklären hat Priv.-Doc. Dr.F.Sejler ca. 15 Fälle von Chlorose theils mit Arsenik, theils mit Eisen, theils mit Eisen und Arsenik in der medicinischen Universitätspoliklinik und in seiner Privatpraxis behandelt und die Fortschritte hinsichtlich Hb. und Erythrocytenneubildung wöchentlich durch entsprechende Bestimmungen controllirt. Herr Dr. Seiler war so freundlich mir seine Krankengeschichten, die aus den Jahren 1907 und 1908 stammen, zu überlassen und hat mich beauftragt die Untersuchungen unter seiner Leitung weiter zu führen. Ich konnte dann auch in den letıten 2 Jahren weitere 20 Fälle in der angeführten Weise behandeln und beobachten und bin nun im Stande über die erwähnte Frage an Hand eines Materials von 33 Fälen zu berichten. Veine Patienten stammten zum grössten Theil aus der medicinischen Universitätspoliklinik, die übrigen verdanke ich der Freundlichkeit von Herrn Professor Sahli, welcher das Material seiner Klinik gütigst zur Verfügung gestellt hat.

Ich stelle im folgenden meine Fälle in drei Gruppen zusammen indem ich diejenigen vereinige, welche 1. mit Arsenik allein, 2. mit Eisen allein, 3. mit Eisen und Arsenik zugleich behandelt worden sind. Die Patientinnen wurden fast alle ambulant behandelt. Während der Zeit der Beobachtungen wurden regelmässig alle 8 Tage, bei einer geringeren Anzahl in etwas grösseren Zeitabständen Hb.-Bestimmungen und Blutkörperchenzählungen nach den üblichen Methoden (Hämometer nach Sahli) ausgeführt. Der Hämoglobinquotient wurde berechnet unter Annahme einer normalen Zahl der Erythrocyten von כ̆ Millionen und eines Hämoglobinwerthes von $70=100 \mathrm{pCt}$. für das weibliche Geschlecht. Es liess sich anf diese Weise zeigen, wie im Laufe der Behandlung bei günstigen Fällen successive die Regeneration von Hämoglobin und Erythrocyton vor sich ging und wie die versehiedenen Behandlungsmethoden in ihrem Werthe bedeutende Untersehiedo erkemnen lassen.

\section{Behandlung der Chlorose mit Arsenik.}

1. Frl. G., $20 \mathrm{~J}$, Anamnese: Menses unregelmässig, manohmal profuse Blutungen, ohne Schmerzen. Frühere Krankheiten: Pneumonie vor 6 Jahren, oft leichte Bronchitis, viel Kopischmerzen. Otitis media purulenta links. Beginn und Symptome der jetzigen Krankheit: nach einjähriger Ueberanstrengung bei der $\Lambda$ rbeit mit Mattigkeit, Ohrensausen, Herzklopfen, Oedeme an den Füssen am Abend nach angestrengter täglicher Arbeit.

Status 7. IX. 09: Allgemeiner körp. u. geist. Habitus normal. Haut und Schleimbäute: Conjunctiven der Lider sind blass, ebenso die Lippen. Gesichtshaut blass. Sinnesorgane: Schwerhörigkeit links; Sehschärfe vermindert, Patientin klagt, dass sie abends mit der Lampe nicht gut arbeiten kann. Respirationsorgane normal. Circulationsorgane: Erweiterung des linken Ventrikels, systolisches Geräusch an der Mitralis, Nonnensausen. Geschlechtsorgane: linke Ovarialgegend ist etwas druckempfindlich, bei der Menstruation aber hat Patientin keine Schmerzen, kein Fluor. 
Behandlung und Untersuchung:

7. IX. 09 Hb. 40 Ther.: Acid. ars. 0,05 ad Pil. $202 \times$ tgl. 2 Pil.

14. IX. $09 \mathrm{Hb}, 40$

$$
\text { Er. } 3,0 \text { Mill. }
$$

21. IX. $09 \mathrm{Hb}, 50$

$$
\text { Er. 3,6 Mill. }
$$

(Acid. ars, 0,01 pro dio)

Therapie gleich

$$
\text { Er. 3,8 Mill. }
$$

Geringer Anstieg des Hb. und der Erythr.-Zahl.

2. Frau H., 28 J. Anamnese: Erkrankung vor einem Jahr nach einer Geburt mit allgemeiner Körperschwäche, Appetitlosigkeit, Schwindel bei Beugen, Kopfweh.

Status 10. IX. 09. Haut und Schleimbäute blass. Vergrösserung des Herzens nach links. Auskultatorisch deutliches Nonnensausen an den Halsvenen hörbar.

Behandlung und Untersuchung:

20. IX. $09 \mathrm{Hb}, 23$ Ther.: Acid. ars. 0,0132 pro die innerlich

Er. 3,6

27. IX. $09 \mathrm{Hb} .21$

Er. 3,5

Allgemeinbefinden ist nicht gebessert.

3. Frl. J, 19 J. Anamnese: Beginn und Symptome der Chlorose: (Patientin hat noch Symptome ron Ulcus ventriculi gehabt) Kopfschmerz, Sehwäche, Müdigkeit, Herzklopfen, Dyspnoe, Menses stark und unregelmässig.

Status 28. VII. 09. Haut und Schleimhäute blass. Patientin macht überhaupt den Eindruck einer schwächlichen, schlecht ernährten Person. Kein Blut im Stuhl.

Bebandlung und Untersuchug:

28. VlI. 09 Hb. 50 Ther.: Acid. ars. in Inject. 0,005 pro die Er. 4,2Mill.

29. VII. $09 \quad n \quad n \quad n \quad n \quad n \quad 0,01 \quad n \quad n$ 31. VII. 09 " $n " n, n, 0075 n$

1. VII. 09 Patientin hat Diarrhoe. Also 0,01 pro die konnte sie nicht vertragen, vielleicht ist das auf ihr Ulous ventrieuli zurückzuführen.

3. VIII. 09 Hb. 50 Ther. Acid. ars. in Inject. 0,0075 pro die Eir. 4,2

4. VIII. 09

5. VIIL, 09

6. VIII. 09

7. VIII. 09

8. VIII. 09

9. VIII. 09

10. VIII. 09

$\begin{array}{lllllllll}\text { Magenschmerz } & n & n & n & n & n & 0,01 & n & n \\ & n & n & n & n & n & 0,005 & " & n \\ \text { Magenschmerz. } & n & n & n & n & n & 0,0075 & n & n \\ & " & n & n & n & n & 0,005 & n & n \\ \text { Hb. 50 } & " & n & n & n & n & 0,005 & n & n \\ \text { Er. 3,8 } & n & n & n & n & n & 0,005 & n & n \\ & & & & & & & \end{array}$

Im Allgemeinen befindet sich die Patientin nicht besser.

4. M. S., 17 J. Anamnese: Beginn und Symptome der Krankheit vor 2 Monaten mit Kopfschmerzen von stechendem Charakter, Sclwwäche, Müdigkeit, Herzklopfen, Dysproe bei raschem Gehen und Aufsteigen. Nenses 3-4 Tage lang, seit 8 Monaten unregelmässig, zuletzt ganz ausgeblieben.

Status. 13. V1I. 09: Hant und Schleimhäute blass. Subjectiy Ohrensausen. Circulationsorgane: Nonnensausen sehr ausgesprochen, mässige Dilatation des linken Ventrikels. Systolisches Geräusch an der Herzspitze. 
Beitrag zur Kenntniss d.Wirkung v. Eisen u. Arsenik als Mittel gegen d.Chlorose. 399

Behandlung und Untersuchung:

13. VII. 09 Hb. 32 Ther.: Acid. ars. 0,1 pil. 30

Er. 2,3 $4 \times$ tgl. 1 Pille $(0,013$ Acid. ars. pro die $)$

21. VII. 09 Hb. 36 Ther.: Acid. ars. 0,12 pil. 40

Er. $2,7 \quad 3 \times$ tgl. 2 Pillen $(0,018$ Acid. ars. pro die $)$

28. VII. 09 Hb. 40 Ther.: Acid. ars. 0,12 pilul. 40

21. $3,5 \quad 3 \times \operatorname{tgl} .2$ Pillen $(0,018$ Acid. ars. pro die)

Die grossen Arsendosen werden gut vertragen, kein Wechsel im subjectiven Allgemeinbefinden.

5. Lina W., 17 J. Anamnese: Mutter leidet an 'Tubercul. pulmonum, eine Schwester anämisch. Seit 1 Jahr fühlte sich die Patientin immer matt, hatte sehr viel Schwindelanfäle und sogar manchmal Ohnmaclitsanfäle. Vor einigen Monaten bemerkte Patientin, dass sie jeden Abend, nachdem sie am Tage viel herumgegangen war, geschwollene Beine hatte, die Oedeme schwanden aber Nachts. Schon seit mehreren Jahren war l'atientin sehr blass gewesen, eine Verschlimmernng des Zustandes trat vor 1 Jahr ein. Patientin hat noch keino Menses.

Status 23. IX. 09: Herz vergrössert, an der Mitralis ein starkes, systolisches Geräusch. Nonnensausen beiderseits.

Behandlung und Untersuchung:

23. IX. 09 IIb. 10

Ther.: Acid, ars. in Inject. 0,002 p.d.

Fr. 3,1

24. - 30. IX. 09

31. IX. 09 IIb. 9

Fr. 2,4

Wegen Misserfolgs muss Arseniktherapie aufgehoben werden.

6. Frl. B., 28 J. Anamnese: Seit 3 Monaten Schwindelanfälle, oft Kopfschmorzen. Seither blasses Aussehen, Hitzegefühl im Kopf, leichte Ermüdbarkeit bei der Arbeit, Herzklopfen und Athemnoth bei 'l'reppensteigen, zeitweise Oedeme in der Knöchelgegend. Sie soll von jeher schwächlich gewesen sein: es habe ihr bald da, bald dort gefehlt, was, weiss sie nicht anzugeben. Menses vor einem Jahre regelmässig; seither bald alle 14 Tage, bald wieder nur alle 2 Monate.

Status vom 4. XI. 09: Kleine, schwächliche Patientin; von mässigem Ernährungszustand, Sensorjum frei. Klagen: Schwäche, Schwindelgefühl, Kopfweh, Herzklopfen, Dyspnoe bei Anstrengung. Puls 92, von geringer Spannung, regelmässig; Blutdruck 150; Respiration 26. Haut und Schleimhäute blass, keine Cyanose, keine Oedeme; leichtes Nonnensausen; über Mitralis systolisches Geräusch.

Behandlung und Untersuchung:

4. XI. 09 Hb. 5,0 Ther.: Acid, ars. in Injection 0,001

5. XI. 09

Er. 4,7 Mill.

6. XI. 09

7. XI, 09

8. XI. 09

9. XI. 09

10. XI. 09

11. XI. 09

12. XI. 09

Hb. 50

Er. 4,9

13. XI. 09

14. XI. 09

15. XI. 09

$\begin{array}{lllll}n & n & n & n & 0,002 \\ n & n & n & n & 0,003 \\ " & n & n & n & 0,004 \\ n & n & n & n & 0,005 \\ n & n & n & n & 0,006 \\ " & n & n & n & 0,007 \\ n & n & n & n & 0,007 \\ & & & & \\ " & n & n & n & 0,008 \\ n & n & n & n & 0,009 \\ n & n & n & n & 0,01 \\ n & n & n & n & 0,01 \\ & & & & 26\end{array}$

Zeitschrift f. exp. Pathologie u. Therapie. S. Ed. 
16. X1. 09

17. XI. 09

18. Xr. 09

19. XI. 09

20. XI: 09

21. XI. 09

22. XI. 09

23. XI. 09

24. XI. 09

25. XI. 09

26. XI. 09

27. XI. 09
Ther.: Acid, ars. in Injection 0,01

Hb. 45

Er. 4,7

Hb. 52

Er. 5,0
$" n, " n \quad 0,01$

$n \quad n \quad n \quad 0,01$

" $\quad$ " $\quad$ " $\quad 0,01$

$\begin{array}{lllll}n & n & n & n & 0,01 \\ " & n & n & n & 0,01\end{array}$

$" n \quad n \quad 0,01$

$\begin{array}{lllll}n & n & n & n & n\end{array}$

$\begin{array}{lllll}n & n & n & n & 0,014\end{array}$

$n \quad n \quad n \quad n \quad 0,015$

" $" \quad$ " $, \quad 0,015$

$\begin{array}{lllll}" & " & " & " & 0,015 \\ " & " & " & n & 0,017\end{array}$

" n n n 0,017

Arsen wurd imemer gut vertragen. Vermehrung des Hb. und der Er.-Zahl gering.

7. Frl. N., 19. J. Anamnese: Patientin menstruirte seit ihrem 12. Jahre unregelmässig, ohne Scbmerzen. Seit dem letzten Jahre keine Menses. Seit 5 Jahren ist die Patientin immer bleich gowesen; eine besondere Verschlimmerung des $\mathrm{Zu}$ standes trat immer im Frühling auf. Sie war sehr schwach und batte viel Kopfweb, auch hatte sie oft Schwindel. Oft Herzklopfen, das bei T'reppensteigen sehr stark wurde und mit Athemnoth verbunden waren.

Status 12. X. 09: Haut und Schleimhäute von gelblicher Farbe. Respirationsorgane normal. Circulationsorgane ausser Nonnensausen normal.

Behandlung und Untersuchung:

12. X. $09 \mathrm{Hb} .40$ Ther.: Acid. ars. in Inject. 0,002 pro die.

$$
\text { Er. } 5,0
$$

13. X. 09 und 14. X. 09

15. X. $09, \quad 16$. X. 09

17. X. 09, 18. X. 09

19. X. 09 Hb. 47

20. X. 09

$$
\text { Er. } 4,5
$$

21. X. 09 und 22. X. 09

23. X. 09

24. X. $09,25$. X. 09

26. X. 09 Hb. 43

$$
\text { Er. } 3,5
$$

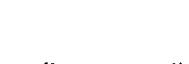

$" n$

$" n$

$n$

$n$

Geringe Vermehrung des Hb.; starke Verminderung der Er.-Zahl.

8. F. H., 19 J. Anamnese: Vor einigen Monaten Beginn der Krankheit mit Mattigkeit, Kopfschmerzen, Schlafsucht, rascher Ermüdung, Ohrensausen, allgemeinem Unwohlsein. Menses seit dem 14. Jahre unregelmässig mit Schmerzen.

Status 18. XI. 09: Mittelgrosses, gut genährtes Mädchen, körperlich und geistig gut entwickelt. Haut blass, Schleimhäute trocken. Circulationsorgane: Verstärkung des 2. Pulmonaltons, keine Geräusche, kein Nonnensausen.

Behandlung und Untersuchung:
18. XI. 09 Hb. 55
Ther.: Acid. ars. 0,05 pil. 20
Er. 5,8
$3 \times$ tgl. 1 Pille $(0,0075$ Acid. ars. p. d. $)$
24. XI. 09
Hb. 65
Acid. ars. 0,05 pil. 20
Er. 5,6
$3 \times$ tgl. 1 Pille $(0,0075$ Acid. ars. p. d.) 
Beitrag zur Kenntniss d. Wirkung v. Eisen u. Arsenik als Mittel gegen d.Chlorose. 401

1. XII. 09 Hb. 65 Ther.: Acid. ars. 0,05 pil. 20

Er. $5,4 \quad 3 \times$ tgl. 1 Pille $(0,0075$ Acid. ars. p. d. $)$

Leichte Verminderung der Er.-\%ahl und Vermehrung des $\mathrm{Hb}$.

9. Frl. H., 17 J. Anamnese: Hrkrankung vor eipigen Monaten mit Sehwäche, Herzklopfen, Kopfschmezzen. Vergrösserung des Herzens nach links, systolisches Geräusch an der Mitralis. Nonnensausen.

Behandlung und Untersuchung:

22. XII. 08 Hb. 31 Ther.: Acid. ars. 0,05 pil. 30 tgl. 4

Er. $2,9 \quad(0,0066$ Acid. ars. pro die $)$

12. J. 09 Hb. 27 " 2 cid. ars. 0,008 pro die

Er. 2,5

Arsenil wird gut vertragen, aber keine Besserung abjectiv und subjectiv.

10. Fil. R. Anamnese: Beginn der Krankheit mit für Chlorose typischen Symptomen. Patientin klagt jetzt hauptsächlich über Kopfschmerzen, Mädigkeit, Herzklopfen und Athemnoth. Herz normal; Nonnensausen sind vorhanden.

Behandlung und Untersuchung:

17. II. 09 Ilb. 27 Ther.: Acid. ars. 0,006 pro die innerlich Er. 4,1

12. III. 09 IIb. 35 Fir. 4,1

Subjectiv leine Besserung trotz dem Hämoglobinanstieg.

11. Frl. B., 191/2 J. Anamnese: Fine Schwester von der Patientin litt ebenfalls an Chlorose. Patientin selbst war schon vor 2 lahren an Chlorose erkrankt und entsprechend behandelt. Beginn der jetzigen Frkrankung im Frühjabr mit Herzklopfen, Mattigkeit, Kopfschmerzen etc.

Status 12. XII, 07: Systolisches Herzgeräusch, an der Spitze maximal; Vergrösserung des Herzens nach links und oben.

Behandlung und Untersuchung:

12. XII. $07 \mathrm{Hb} .35$ Ther.: Acid. ars. 0,004 pro die innerlich

13. XII. 07 Er. 4,0

14. XII. 07

15. XII. 07 bis 24 . XII. 07

24. XII. 07 Hb. 35

Er. 3,6

Behandlung obne Erfolg.

12. Fri. W., 21 J. Anamnese: Erkrankung vor ca. 2 Monaten mit Schwäche, Herzklopfen, Scblafsucht, Kopfschmerzen, Magenbeschwerden, allgemeinem Unwohlsein. Früher war Patientin immer gesund. Familienanamnese ohne Belang.

Status 13. II. 08: Patientin zeigt keine Herz- und Venengeräusche.

Behandlung und Untersuchung:

13. II. 08 IIb. 35 Ther.: Acid. ars. 0,006 pro die innerlich

27. II, $08 \mathrm{Hb}, 35$

Br. 4,0 Diät wegen Magenbesehwerden

Er. 3,6 Magenbeschwerden sind gebessert.

Blutbild ist eher schlechter geworden.

13. Frl. Z., 18 J. Anamnese: Beginn der Krankheit mit läufigen Kopfschmerzen, Herzklopfen, Schwindelanfällen, Hyperaciditätsbeschwerden. 
Status 5. 111. 08: Am Herzen schwaches systolisches Geräusch an der Pulmonalis, Nonnersausen. Aussehen sehr blass und matt.

Behanding und Untersachung:

5. 11I. 08 Hb, 39 Ther.: Acid. ars. 0,008 pro die innerlich

14. III. $08 \mathrm{Hb}, 47$

Er. 4,7
$\mathrm{Hb} .47$
Er. 4,6

Subjectiv fühlt sich Patientin nicht besser. Anstieg des Hämoglobins.

Im Anschluss an diese Krankengeschichten stelle ich im Folgenden die Resultate tabellarisch zusammen:

Tabelle I. Resultate der Behandlung mit Arsenik.

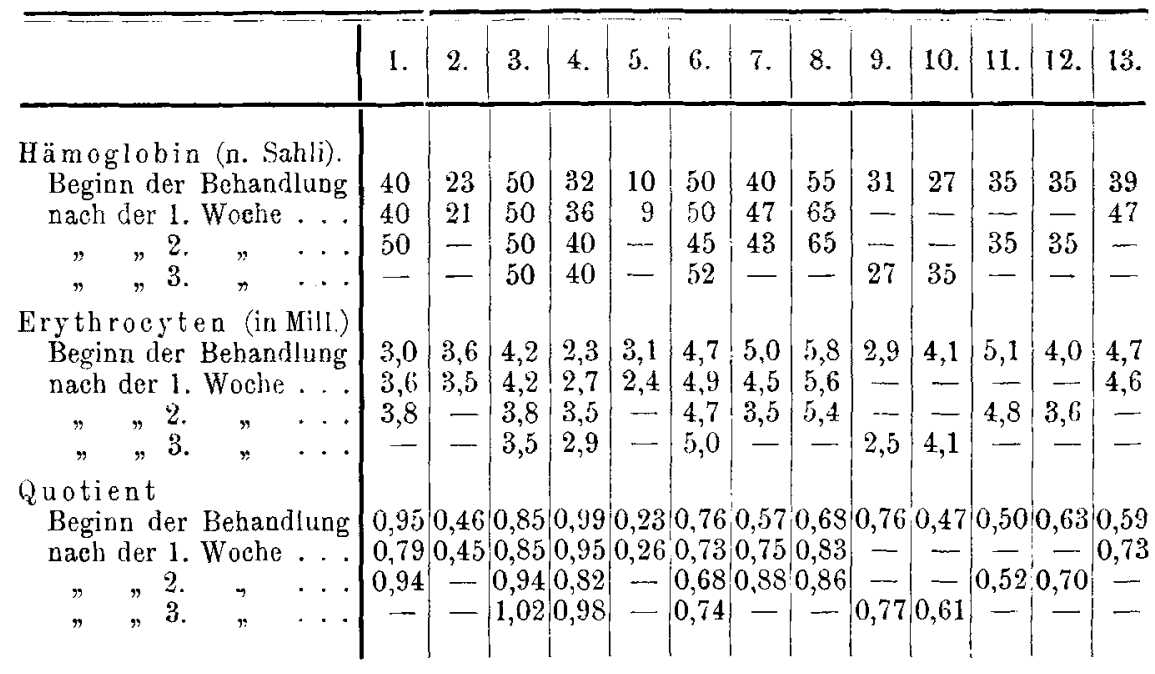

In der folgenden Tabelle Il habe ich die Durchschnittswerthe bei Beginn und zu Ende der Behandlung zusammengostellt, wobei ich diejenigen Fälle zusammenfasste, welche während eines gleichen Zeitraums behandelt worden sind.

Tabelle II. Durchschnittsresultate bei Behandlung mit Arsenik ${ }^{1}$ ).

\begin{tabular}{|c|c|c|c|c|c|}
\hline & & & \multicolumn{3}{|c|}{ Dauer der Behandlung. } \\
\hline & & & 1 Woche & 2 Wochen & 3 Wochen \\
\hline Hämoglobin: Beginn & $\operatorname{der}$ & Behandlung & $\begin{array}{l}36,50 \\
35,00\end{array}$ & $\begin{array}{l}41,00 \\
45,60\end{array}$ & $\begin{array}{l}38,00 \\
40,80\end{array}$ \\
\hline Erythrocyten: Beginn & $"$ & $"$ & $\begin{array}{l}3,35 \\
2,95\end{array}$ & $\begin{array}{l}4,42 \\
4,22\end{array}$ & $\begin{array}{l}4,04 \\
3,60\end{array}$ \\
\hline Quotient: $\begin{aligned} \text { Beginn } \\
\text { Ende }\end{aligned}$ & $"$ & $"$ & $\begin{array}{l}0,35 \\
0,35\end{array}$ & $\begin{array}{l}0,67 \\
0,78\end{array}$ & $\begin{array}{l}0,77 \\
0,82\end{array}$ \\
\hline
\end{tabular}

Aus diesen Untersuchungen geht hervor, dass die reine Arsentherapie bei der Chlorose keine Erfolge aufzuweisen hat. Nach 2-3wöchiger

1) Die Tabellen sind so bergestellt, dass immer gleicbartige Fälle zusammengefasst wurden. 
Behandlung zeigte zwar das Hämoglobin im Durchschnitt eine ganz leichte Vermehrung, die Zahl der Erythrocyten dagegen nahm in der Mehrzahl der Fälle merklich ab.

Es liess sich somit, wenigstens was die Chlorose betriff, ein Einfluss des Arseniks auf die Funktion des Knochenmarks, rothe Blutkörperchen zu bilden, in keiner Weise feststellen. Die reine Arseniktherapie, d. h. die Anwendung des Arseniks unter Ausschluss hygienischdiätetischer Vorschriften (Liegekur, Höhenaufenthalt, Hydrotherapie etc.) ist absolut erfolglos. Die Erfolge, welche durch Trinkkuren von arsenikhaltigen Wässern an den bekannten Kurorten bei Chlorose zweifellos erzielt werden, beruhen also wohl fast ausschliesslich auf der Wirkung des Klimas, der veränderten Lebensweise ete. und nicht auf der Wirkung des Arseniks. Es sei denn, dass es sich um Wässer mit gleichzeitigem Arsen- und Eisengehalt handelt. Ich möchte nicht unterlassen, bei dieser Gelegenheit die Erfahrung zu betonen, dass Chlorosekranke im Allgemeinen nur mässige Dosen von Arsenik vertragen, so dass z. B. von unseren Fällen mehrere schon auf Mengen von 0,006-0,01 Acid. arsenic. pro die mit intensivem Unwohlsein, Erbrechen, Darmstörungen etc. reagirt haben.

\section{Behandlung der Chlorose mit Eisen.}

1. Frl.S., 17 J. A namnese: Systol. Herzgeräusch, Nonnensausen ist vorbanden. Klagen: Dyspnoe, Herzklopfen, Müdigkeit, Schlafsucht, Schwindelanfälle, Nasenblutungen. Patientin ist klein, mager und hat blasses Aussehen.

Behandlung und Untersuchung:

4. VIII. $09 \mathrm{Hb} .40$ Ther.: Ferri sulfur.

Er. 2,9 Kalii carbon. ana 0,9 pro die innerlich ${ }^{\mathbf{1}}$ )

18. VIII. $09 \mathrm{Hb} .62 \quad, \quad, \quad, \quad, 0,9, \quad$,

25. VIII. $09 \mathrm{Hb} .65 \quad, \quad " \quad " \quad, 0,9, n$,

1. IX. $09 \mathrm{Hb} \cdot \mathbf{H 0} \quad, \quad " \quad$ " $7,0,9, \quad$,

Er. 4,8

Hämoglobinwerth $\frac{70}{70}=$ Norm für weibliches Gesehlecht $=100 \mathrm{pCt}$ :

Patientin füblt sich viel besser. Keine accidentelle Geräusche und Nonnensausen mehr. Geheilt entlassen.

2. Frl. J., 25 J. Anamnese: Erkrankung vor 6 Monaten mit Kopfscbwindel, Dyspnoe, Schwäche. Patientin bietet jetzt die typischen Chlorosesymptome dar.

Behandlung und Untersuchung:

17. VIIl. 09 Hb. 50 Ther.: Ferri sulfur.

Er. 3,5 Kalii carbon. ana 0,6 pro die innerlich

1) Wir verordnen in der Regel:

Rp. Ferri sulfurici

Kalii carbonici ana 10,0

Mas. pil. q. s. ut f. pil. No. C.

S. $3 \times$ tägl. 2-3 Pillen zu nehmen, 
24. VIII. $09 \mathrm{Hb} .55$ Ther.: Ferri sulfur.

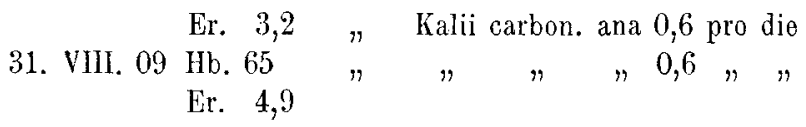

Subjectiv fühlt sich Patientin besser. Blutbild ist wesentlich gebessert. Patientin ist geheilt entlassen.

3. Frl. H., 27 J. Anamnese: Mutter und 2 Geschwister waren anämisch. Schon als Kind war Patientin immer blass und schwächlich. Jetzt vor 1 Jahre haben die Erscheinungen zugenommen. Patientin klagt über Kopfweh, Dyspnoe, Schwäche etc.

Behandlung und Untersuchung:

14. IX. 09 Hb. 21 Ther.: Ferri sulfur.

Er. $3,5 \quad$ Kalii carbon. ana 0,9 pro die innerlich

24. IX. 09 Hb. $35 \quad " \quad$ " " 0,9 " "

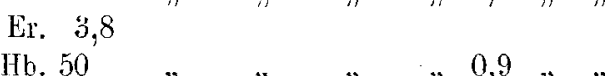

1. X. $09 \mathrm{Hb}, 50$

Er. 4,0

7. Х. 09 Hb. 58

Er. 4,1

14. X. 09 Hb. 65 Er. 5,5

Patientin fühlt sich ganz gut. Hb.- und Er.-Zahl sind rasch angestiegen.

4. Frl. G., 21 J. Anamnese und Status für eine Chlorose typisch. Patientin bietet noch Symptome von Ulcus ventriculi dar.

Behandlung und Untersuchung:

\begin{tabular}{|c|c|c|c|c|c|c|c|}
\hline 21. IX. 09 & $\begin{array}{l}\text { Hb. } 50 \\
\text { Er. } \quad 3,8\end{array}$ & $\begin{array}{c}\text { Ther.: } \\
n\end{array}$ & $\begin{array}{l}\text { Ferr. sulfur. } \\
\text { Kal. carbon. }\end{array}$ & $\begin{array}{l}0,9 \\
\text { ana }\end{array}$ & & roo & ie innerlich \\
\hline 28. IX. 09 & $\begin{array}{l}\mathrm{Hb} .55 \\
\text { Er. } \quad 4,1\end{array}$ & $"$ & $n$ & $"$ & 0,9 & $"$ & $"$ \\
\hline 5. X. 09 & $\begin{array}{l}\text { Hb. } 65 \\
\text { Er. } \quad 4,0\end{array}$ & $"$ & $n$ & $"$ & 0,9 & $"$ & $"$ \\
\hline 12. X. 09 & $\begin{array}{l}\text { Hb. } 70 \\
\text { Er. } \quad 4,2\end{array}$ & $n$ & $n$ & $"$ & 0,9 & $"$ & $"$ \\
\hline
\end{tabular}

Regelmässige Steigerung des Hämoglobins. Keine grosse Vermehrung der Er.Zahl. Hb. ist bis zur Norm gestiegen. Keine Chlorosebeschwerden mehr.

5. Frl. B., 27 J. An amnese: Unregelmässige Menstruation seit ihrem 18. Jahre, vor einem Jahre ganz aufgehört. Klagen über Kopfweh, Ohrensausen, Müdigkeit, Dyspnoe, Schlafsucht. Herz: Dilatation des linken Ventrikels, systolisches Geräusch an der Spitze maximal. Respirationsorgane normal. Nonnensausen beiderseits an den Halsvenen. Haut und Schleimhäute sind trocken aber nicht sehr blass.

Behandlung und Untersuchung:

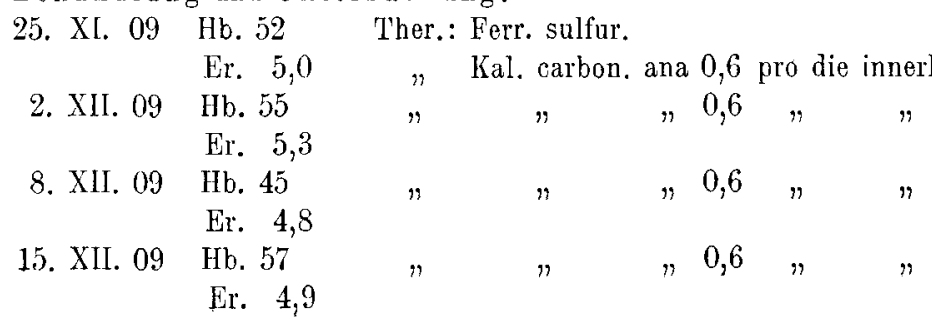


Beitrag zur Kenntniss d.Wirkung v. Eisen u. Arsenik als Mittel gegen d Chlorose. 405

22. XII. 09 Hb. 68 Ther.: Ferr. sulfur.

29. XII. 09 Er. $4,9 \quad " \quad$ Kal. carbon. ana 0,6 pro die innerlich

Er. 5,5

Patientin fühlt sich ganz gut. Blutbild ist fast normal.

6. Frl. F., 19 J. Anamnese: Die Symptome der Chlorose hat die Patientin schon seit 3 Jahren, ist aber nie entsprechend behandelt. Menses seit ihrem 15. Jahre unregelmässig und nicht stark. Herz normal; kaum hörbares Nonnensausen. Respirationsorgane normal.

Behandlung und Untersuchung:
2. X. 09 Hb. 47
Ther.: Ferr. sulfur.
Er. 4,6
$"$
Kal. carbon. ana 0,9 pro die innerlich
16. X. 08 Hb. 54
Er. 4,4
$" \quad, 0,9 \quad " \quad$,

Patientin ist nicht mehr gekommen.

7. Frl. L., 17 J. Anamnese: Vor 2 Monaten verspürte die Patientin Müdigkeit, Herzklopfen und war dann den ganzen Tag schläfrig. Status: TypischeChlorose.

Behandlung und Untersuchung:
3. II. $10 \mathrm{Hb}, 40$
Er. 5,0
Ther.: Ferr. sulfur.
10. II. 10 Hb. 55
Er. 5,0
17. II. 10 Hb. 60
Er. 5,3
24. II. 10 Hb. 66
Er. 5,9
" Kal. carbon. ana 0,9 pro die innerlich

Zahl der Er. normal. Patientin ist geheilt entlassen.

8. Frau W., 25 J. A namnese: Seit einem Jahre bleichsüchtig. Nonnensausen stark. Im Stehen systolisches Geräusch an der Mitralis. Klagen: Sohlafsucht, Müdigkeit, Schwindelanfälle, Nasenblutungen. Hant und Schleimhäute sind sehr blass.

Behandlung und Untersuchung:
13. XI. 09 Hb. 31
Ther.: Ferr. sulfur.
Er. 4,
$"$ Kal. carbon. ana 0,6 pro die innerlich
26. XI. 09 Hb. 47
10. XII. 09 Hb. 54
Er. 6,2

9. Frl. R., M. 21 J. Anamnese: Erkrankung vor einem Jahr mit Kopfschmerzen, Athemnoth, Müdigkeit, Ohrensausen, Schlafsucht, Abends mit Oedemen an Händen und Füssen. Kurz vor der Erkrankung musste die Patientin schwere körperliche Arbeit leisten. Familienanamnese ohne Belang. Typische Chlorose.

Behandlung und Untersuchung:
1. I. $09 \mathrm{Hb} .35$
Ther.: Ferr. sulfur.
14. I. 09 Hb. 50
Er. 4,0
28. I. 09 Hb. 62
12. II. $09 \mathrm{Hb} .70$
" Kal. carbon. ana 0,9 pro die innerlich
Hb. 70
Er. 5,3

n

$$
n
$$
n

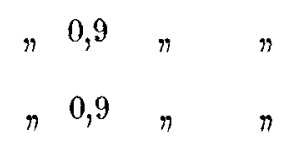


Patientin fühlt sich ganz gesund. Oedense sind auch nicht mehr vorbanden. Blutbild hat sich wesentlich gebessert.

10. Frl. S., 16 J. Anamnese: Patient bietet alle Symptome der Chlorose dar.

Behandlung und Untersuchung:

\begin{tabular}{|c|c|c|c|c|c|c|c|c|}
\hline 12. XI. 07 & $\begin{array}{l}\text { IIb. } 23 \\
\text { Er. } 4,0\end{array}$ & $\begin{array}{c}\text { Ther.: } \\
\text { " }\end{array}$ & $\begin{array}{l}\text { Ferr, sulfur. } \\
\text { Kal. carbon. }\end{array}$ & ana & 0,9 & pro & die & nner \\
\hline 26. XI. 07 & $\begin{array}{l}\text { Hb. } 50 \\
\text { Fr. } 4,6\end{array}$ & $n$ & $\eta$ & $n$ & 0,9 & $n$ & & $n$ \\
\hline 10. XII. 07 & $\begin{array}{l}\text { IIb. } 50 \\
\text { E: } \quad 5,0\end{array}$ & $"$ & $n$ & $n$ & 0,9 & $n$ & & $n$ \\
\hline 31. XII. 07 & $\begin{array}{l}\text { Hb. } 58 \\
\text { Er. } 5,1\end{array}$ & $n$ & $n$ & $n$ & 0,9 & $\eta$ & & $"$ \\
\hline
\end{tabular}

Die Pillen werden gut vertragen. Hämoglobin steigt rasch an, Er.-Zahl ebenfalls.

11. Frl. B., 19 3. Anamnese: Seit einem Jahr fühlte sich die Patientin zeitweise matt, schwach, schläfrig, hatte Kopfweh etc., bald gingen die Erscheinungen zurück, bald traten sie aber von neuem anf. Respirationsorgane und Herz normal, Nonnensausen sehr ausgesprochen. Menses normal, seit ihrem 14. Jahr. Patientin hat Spuren Eiweiss im Urin, ödematöses Gesicht und Oedeme an den unteren Extremitäten.

Behandlung und Untersuchung:

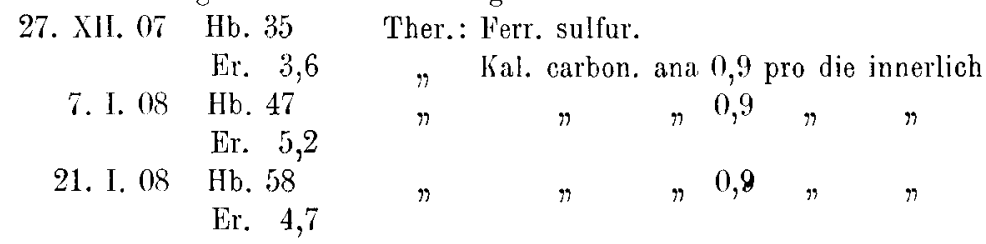

Rascher Anstieg des Hämoglobins. Subjectiv fühlt sich Patientin besser.

12. Frl. S. Symptom der Chlorose sind von solchen eines Ulcus ventriculi bogleitet. l'atientin sieht schlecht aus, hat wenig Appetit. Ulcusbeschwerden seit 2 Jahren. Chlorose seit einem falur mit cbarakteristischen Erscheinungen.

Behandlung und Untersuchung:

19. XI. 07 Hb. 31 Ther.: Ferr. sulfur.

Er. 4,1 "Kal. carbon. ana 0,6 pro die innerlich

26. XI. 07 Hb. $50 \quad$ E.r. $4,2 \quad " \quad$ " $0,6 \quad " \quad$ "

Patientin muss verreisen, darum kan sie nicht weiter behandelt werden.

In den nebenstehenden Tabollen III und IV stelle ich die Resultate der Behandlung mit Eisen einzig bei 12 chlorosekranken Patientinnen zusammen.

Es geht aus diesen Zusammenstellungen sehr deutlich hervor, dass unter der Behandlung mit Bisen allein eine prompte Besserung und Heilung der Chlorose in den meisten Fällen erzielt wird. Wir werden nun im folgenden sehen wie sich die Chlorose unter der Behandlung mit Eisen + Arsenik verhält. 
Beitrag zur Kenntniss d.Wirkung v. Eisen u. Arsenik als Mittel gegen d. Chlorose. 407

Tabelle 111. Resultate der Behandlung mit Blaud'sehon Pillen.

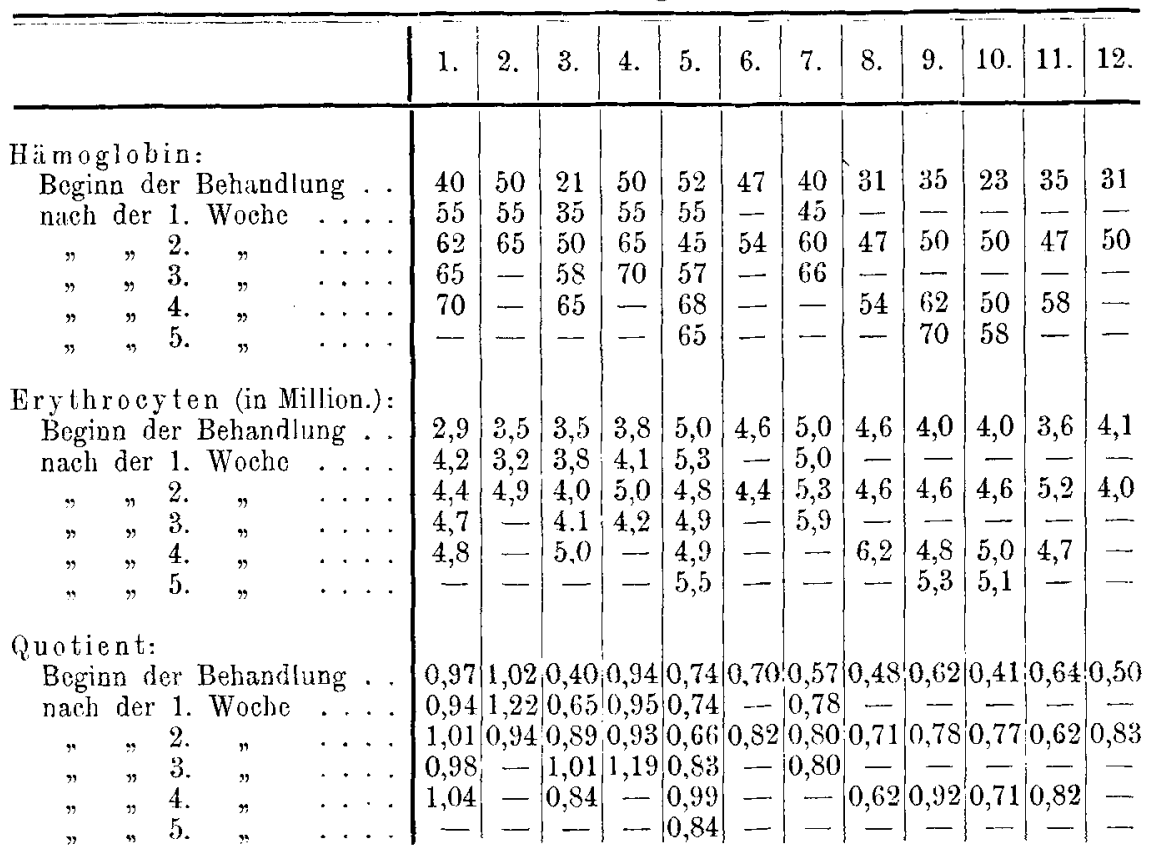

Tabelle IV. Durchschnittsresultate bei Bchandlung mit Blaud'schen Pillen $\mathbf{1}$.

\begin{tabular}{|c|c|c|c|c|c|c|c|}
\hline & & & & I Wochen & $\begin{array}{l}\text { Dauer der I } \\
3 \text { Wochen }\end{array}$ & $\begin{array}{l}\text { Behandlung } \\
4 \text { Wochen }\end{array}$ & 5 Wochen \\
\hline Hämoglobin: & $\begin{array}{l}\text { Beginn } \\
\text { Ende }\end{array}$ & der & Behandlung & $\begin{array}{l}42,00 \\
56,00\end{array}$ & $\begin{array}{l}45,00 \\
68,00\end{array}$ & $\begin{array}{l}32,00 \\
62,00\end{array}$ & $\begin{array}{l}37,00 \\
64,00\end{array}$ \\
\hline Erythroeyton: & $\begin{array}{l}\text { Beginn } \\
\text { Fnde }\end{array}$ & $"$ & $"$ & $\begin{array}{l}4,06 \\
4,43\end{array}$ & $\begin{array}{l}4,40 \\
5,00\end{array}$ & $\begin{array}{l}3,65 \\
5,30\end{array}$ & $\begin{array}{l}4,30 \\
5,30\end{array}$ \\
\hline Quotient: & $\begin{array}{l}\text { Perinn } \\
\text { Ende }\end{array}$ & $"$ & $"$ & $\begin{array}{l}0,74 \\
0,86\end{array}$ & $\begin{array}{l}0,75 \\
1,00\end{array}$ & $\begin{array}{l}0,60 \\
0,83\end{array}$ & $\begin{array}{l}0,74 \\
0,84\end{array}$ \\
\hline
\end{tabular}

\section{Behandlung der Chlorose mit Eisen + Arsenik.}

1. Frl. W. 1,17 J. Anamnese: Eine Schwester von der Patientin leidet auch an Chlorose, andere an Tuberc. pulmonum. Patientin selbst ist vor einem Jahre erkrankt nach schwerer liörperlicher Arbeit mit Kopfschmerzen, Schwinde]anfällen, Müdigkeit, Ohrensausen. Verschlimmernng des Zustandes trat ganz allmählich ein, bis die Patientin sich ganz kiank und arbeitsunfähig fühlte. Jetzt sieht sie sehr schlecht aus, klagt über Herzklopfen, Kopfschmerzen und Müdigkeit. Haut und Schleimhäute sind sehr blass, Zunge belegt. 'I'yp. Status hochgradiger Chlorose.

Behandlung und Untersuchung:

1. X. 09-7. X. 09 Hb. 9 Ther.: Ferr. sulfur., Kal. carbon. ana 0,4 Er. $2,4+\Lambda$ cid. arsen. 0,006 pro die innerlich

7. X. $09-14$. X. $09 \mathrm{Hb} .28 \quad "$ Ferr. sulf., Kal. carbon. ana 0,9 Er. $2,7+$ Acid. arsen. 0,006 pro die

1) cf. Anmerkung S. 402. 


\begin{tabular}{|c|c|c|c|}
\hline \multirow{2}{*}{$\begin{array}{l}\text { 14. X. } 09 \\
\text { 22. X. } 09\end{array}$} & $\mathrm{Hb} .40$ & \multicolumn{2}{|c|}{ Therapie gleich } \\
\hline & $\begin{array}{r}\text { Hb. } 55 \\
\text { Er. } 4,0\end{array}$ & $n$ & $"$ \\
\hline 29. X. 09 & $\begin{array}{r}\text { Hb. } 62 \\
\text { Er. } 4,1\end{array}$ & $"$ & $"$ \\
\hline 5. XI. 09 & $\begin{array}{l}\mathrm{Hb} . \quad 65 \\
\text { Er. } 4,9\end{array}$ & $n$ & $"$ \\
\hline 12. XI. 09 & $\begin{array}{c}\text { Hb. } \quad 68 \\
\text { Er. } 5,1\end{array}$ & $n$ & $"$ \\
\hline 19. XI. 09 & $\begin{array}{c}\text { Hb. } 70 \\
\text { Er. } 5,1\end{array}$ & $n$ & $"$ \\
\hline
\end{tabular}

Prompte Besserung des Blutbildes und gutes Aussehen.

2. Frl. N. Anamnese: Patientin hat typische Symptome der Chlorose, sonst keine Beschwerden.

Behandlung und Untersuchung:

26. X. 09 Hb. 43 Ther.: Acid. arsen. 0,003

$$
\text { Er. 3,5 } \quad+\text { Ferr. sulf. }
$$

2. XI. 09 Hb. $46 \quad$ Kal. carbon. ana 0,6 pro die innerlich

Er. $4,0 \quad \because \quad$ Acid. arsen. 0,0045

9. XI. 09 Hb. $58 \quad+$ Ferr. sulf. 0,9

Eir. 5,0 Kal. carbon. ana 0,9 , ,

16. XI. 09 Hb. 65 Therapie wie am 9. XI. 09

23. XI. 09 Hb. $70 \quad$ \# $\quad$ " 9. XI. 09 Er. 5,5 bessert.

Patientin fühlt sich ganz gut; ist geheilt entlassen. Blutbild hat sich rasch ge-

3. Frl. J, 18 J. Anamnese: Patientin hat Lungenkatarrh, der auf Tbe verdächtig ist. Menses seit 2 Jahren unregelmässig mit Schmerzen; menstrueller Blutverlust ist sehr gering. Keine frühere Krankheiten. Beginn und Symptome der jetzigen Krankheit: vor 2 Jahren mit Schwäche, Kopfschmerzen, Müdigkeit, Herzklopfen. Tbc. in der Familie ist nicht vorhanden. Herzgeräusch, Nonnensausen.

Behandlung und Untersuchung:

25. IX. 09 Hb. 25 Ther.: Ac. ars. 0,003 + Ferr. sulfur.

Er. 3,2 Kal. carb. ana 0,9 pro die innerlich

2. X. $09 \mathrm{Hb} .35$

Er. 3,6

9. X. 09 Hb. 40 'therapie gleich.

Er. 3,7

15. X. 09 Hb. 50

Er. 4,5

23. X. 09 Hb. 60

Er. 4,6

30. X. 09 Hb. 70

Er. 5,0

5. XI. 09 Hb. 75

Er. 5,1

Objectiv und subjectiv gehts der Patientin sehr gut; sie ist geheilt entlassen.

4. Frl. S., 21 J. Anamnese: Beginn und Symptome der Chlorose schon vor 2 Jahren mit Kopfschmerzen, Müdigkeit, Arbeitsunlust. 
Beitrag zur Kenntniss d.Wirkung v. Eisen u. Arsenik als Mittel gegen d. Chlorose. 409

Status den 19. XI. 09: Haut und Schleimhäute blass und trocken, Ernährungszustand gut.

Behandlung und Untersuchung:

19. XI. 09 Hb. 22 Therapie: Acid. arsen. 0,006

Er. $2,2 \quad+$ Ferr. sulf.

25. XI. 09 Hb. 30 Therapie gleich.

Kal, carb. ana 0,72 pro die innerlich

$\begin{array}{lrlll}\text { 2. XII. } 09 & \text { Er. } 3,1 \\ \text { Hb. } 48 & & & \\ \text { Er. } 3,6 & & \\ \text { 9. XII. } 09 & \text { Hb. } 55 & & \\ & \text { Er. } 4,1 & & \\ \text { 16. XII. } 09 & \text { Hb. } 65 \\ & \text { Er. } 5,4 & & \end{array}$

Patientin fühlt sich ganz gut, ist gebeilt entlassen.

5. Frl. J. M., 16 J. Anamnese: Icterus vor einem Jahr. Beginn der Menstruation vor 2 Jahren, bis jetzt nur dreimal anregelmässig und nur wenig. Beginn und Symptome der jetzigen Krankheit: vor $1 \frac{1 / 2}{2}$ Jahren mit Müdigkeit, Kopfschmerzen, Schwindelanfällen, Herzklopfen.

St atus praesens den 22. IV. 10; Ernäbrungszustand gut; Haut und Schleimhäute sehr blass; Herz normal; an den Halsvenen Nonnensausen.

Behandlung und Untersuchung:

22. IV. 10 Hb. 32 Therapie: Acid. arsen. 0,006

Er. 2,6 + Ferr. sulf.

30. IV. $10 \mathrm{Hb}, 45 \quad$ Kal. carbon. ana 0,6 pro die innerlich Er. 3,6 Therapie gleich.

7. V. 10 Hb. 60 " "

Er, 4,2

14. V. $10 \mathrm{Hb}, 70$

Er. 5.2

20. V. 10 Hb. 75

Er. 5,5

Prompter Anstieg des Hb.- und der Er.-Zahl. Patientin ist geheilt entlassen.

6. Frl. M. R., 18 J. Anamnese: Menses vom 13. Jahre an, unregelmässig, seit 8 Monaten keine mehr. Beginn und Symptome dex Chlorose: vor einigen Monaten mit Schwäche, Kopfschwindel, Müdigkeit, Appetitlosigkeit, Herzklopfen.

Status 12. X. 09: Aussehen ist blass. Herz: leichte Dilatation nach links, systolisches Geräusch an der Spitze; Nonnensausen. Patientin leidet zudem an chronischer Obstipation.

Behandlung und Untersuchung:

12. X. 09 Hb. 25 Therapie: Natr. arsenicic. 0,04

Er. 3,8 Ferr. eitr. oxydulat. cum ammon. citr. 1,0

Aq. des, ster. ad 20,0

Zusatz: Thymolkryställchen q. s. ad sterilisat.

13. X. 09 Inject. $1 / 2 \mathrm{ccm}$ obiger Lösung

14. X. 09

$n 1 / 2 n \quad n$

15. X. 09

16. X. 09

17. X. 09 Hb. 25

Er. 3,8

$\begin{array}{lcll}n & 1 / 2 & n & " \\ " & 1 / 2 & n & n \\ " & 3 / 4 & n & n \\ " & 3 / 4 & n & n \\ " & 1 & n & n\end{array}$


18. X. 09

19. X. 09 Magenbeschwerden

20. X. 09

21. X. 09

22, $x, 09$

23. X. 09

24. X. 09

25. X. 09-29. X. 09

29. X. $09 \mathrm{Hb}, 37$

Er. 4,0

30. X. 09-4. XI. 09

4. XI. 09 Inb. 45

Er. 4,2

5. XI. 09-10. XI. 09

10. XI. $09 \mathrm{Hb} .55$

kr. 4,4

11. XI. 09-18. XI, 09

18. XI. 09 Hb. 62

Er. 4,6

19. XI. 09-23. XI. 09

23. Xl. 09 Hb. 65

Hir. 4,8
Inject. $1 \mathrm{ccm}$

$\begin{array}{ccc}" & 1 & \\ " & 3 / 4 & " \\ " & 3 / 4 & " \\ " & 3 / 4 & " \\ " & 3 / 4 & " \\ " & 1 & " \\ " & 1 & " \\ " & 1 & n\end{array}$

$n 13$

$" 1$,

$" 1 "$

$n 1 "$

$" 1 n$

$n 1 n$

$" 1 \%$

Nicht sehr prompter Anstieg des $\mathrm{Hb}$; deutliche Zunahme der Erythrocyten.

7. Frl. F., 22. J. Anamnese: 'Typische Beschwerden der Chlorose bei blühendem Aussehen. Systolisches Geräusch an der Spitze, starkes Nonnensausen.

Behandlung und Untersuchung:

16. IV. 08 IIb. 23 Ther.: Acid. ars. 0,006

Er. 3,6 + Ferr. sulf.

Kal. carbon. ana 0,6 pro die innerlich

27. 1V. 08 Hb. 35 Therapie gleich

Er. 5,0

11. V. $08 \mathrm{Hb} .58$

Fr, 5,0

Keine Beschwerden mehr; Patientin fühlt sich gut.

8. Frl. J., 24 J. Anamnese: Schon einmal vor 5 Jahren überstandene Chlorose. Auf die Behandlung, die Patientin nicht angeben kann, gingen die Beschwerden innerhalb 6 Wochen ganz zurück. Jetzt Erkrankung vor 6 Monaten mit Herzklopfen, A themnoth, Schwindel, Müdigkeit, Blässe.

Behandlung und Untersuchung:

24. III. 08 Hb. 39 Ther.: Acid. arsen, 0,006

Er. 4,8 + Ferr. sulfur.

5. IV. $08 \mathrm{Hb} .47 \quad$ Kal. carbon. ana 0,9 pro die innerlich Er. 5,0

Patientin ist nicht mehr zur Untersuchung gekommen.

Auch die Resultate dieser combinirten Behandlung mit Eisen + Arsenik wird in analoger Weise, wie früher, in Tabellenform zusammengestellt. 
Beitrag zur Kenntniss d.Wirkung v. Eisen u. Arsenik als Mittel gegen d. Chlorose. 411

Tabelle V. Resultate der Behandlung mit Eisen + Arsenik.

\begin{tabular}{|c|c|c|c|c|c|c|c|c|c|c|c|c|}
\hline & & & & & 1. & 2. & 3. & 4. & 5. & 6. & 7. & 8. \\
\hline \multicolumn{13}{|c|}{ Hämoglobin: } \\
\hline & n der Beha & & & • & 9 & 43 & 25 & 29 & 32 & 25 & 28 & 39 \\
\hline nach & der 1 . Woch & & & & $\begin{array}{l}28 \\
40\end{array}$ & $\begin{array}{l}46 \\
58\end{array}$ & & & & $\begin{array}{l}25 \\
37\end{array}$ & 32 & 47 \\
\hline$"$ & $\Rightarrow \quad 2$. & & & . & 55 & 65 & 5 & 5 & 70 & 45 & 58 & - \\
\hline & $" 4$. & & & & 62 & $=70$ & 60 & 65 & & & - & - \\
\hline$"$ & $" 5 . \quad " \quad$ & & & & 65 & - & 70 & - & - & 65 & - & - \\
\hline \multirow{2}{*}{\multicolumn{13}{|c|}{$\begin{array}{l}\text { Erythrocyten: } \\
\text { Begin der Behandlung }\end{array}$}} \\
\hline & & & & & 2,4 & 3,5 & 3,2 & 2,2 & 2,6 & $\begin{array}{l}3,8 \\
, 8\end{array}$ & 3,6 & 4,8 \\
\hline & der 1. Woche . & & & & $\begin{array}{l}2,7 \\
47\end{array}$ & $\begin{array}{l}4,0 \\
5,0\end{array}$ & $\begin{array}{l}3,6 \\
3,7\end{array}$ & $\begin{array}{l}3,1 \\
3,6\end{array}$ & $\begin{array}{l}3, \\
4\end{array}$ & $\begin{array}{l}3,8 \\
4,0\end{array}$ & 5,0 & 5,0 \\
\hline & $\Rightarrow \quad 2$. & & & & 4,0 & 5,5 & 4,5 & $\begin{array}{l}4,0 \\
4,1\end{array}$ & $\check{5}, 2$ & $\begin{array}{l}+, 0 \\
4,2\end{array}$ & $\overline{5,0}$ & - \\
\hline " & $\Rightarrow 4$. & & & & 4,4 & 5,5 & 4,6 & 5,4 & 5,5 & 4,4 & - & - \\
\hline$”$ & $” 5$ & & & & 4,9 & - & 5,0 & - & - & 4,6 & - & - \\
\hline \multicolumn{13}{|c|}{$\begin{array}{l}\text { Quotient: } \\
\text { Beginn der Behandlung }\end{array}$} \\
\hline Begin & a der Behandlun & & & & 0,26 & 0,87 & 0,55 & 0.71 & 0,88 & 0,48 & 0,42 & 0,59 \\
\hline nacl & der 1. Woch & & & & 0 , & 0,82 & 0,69 & 0,69 & 0,89 & 0,48 & 0,40 & 0,73 \\
\hline " &, 2 & & & & & 0,8 & 0,77 & 1,31 & 1,02 & 0,66 & - & -- \\
\hline$"$ & $" 3$. & & & & 0 & 0,84 & 0,79 & 0,95 & 0,96 & 0,76 & 0,83 & - \\
\hline$"$ & $\begin{array}{llll}4 . & 5\end{array}$ & & & & $\begin{array}{l}1,08 \\
0,94\end{array}$ & 0,91 & $\begin{array}{l}0,93 \\
1,00\end{array}$ & $0, \varepsilon 6$ & 0,98 & $\begin{array}{l}0,89 \\
100\end{array}$ & $=$ & E \\
\hline
\end{tabular}

Tahelle VI. Durchschnittsresultate bei Behandlung mit Eisen + Arsenik.

\begin{tabular}{|c|c|c|c|c|c|c|c|}
\hline & \multicolumn{4}{|c|}{ Dauer der Behandlung } \\
\hline & & & & 2 Wochen & 3 Wochen & 4 Wochen & 5 Wochen \\
\hline Hämoglobin: & $\begin{array}{l}\text { Beginn } \\
\text { Ende }\end{array}$ & der & $\begin{array}{c}\text { Behandlung } \\
"\end{array}$ & $\begin{array}{l}26,00 \\
\mathbf{4 7}, 00\end{array}$ & $\begin{array}{l}25,00 \\
58,00\end{array}$ & $\begin{array}{l}26,00 \\
64,50\end{array}$ & $\begin{array}{l}19,00 \\
66,00\end{array}$ \\
\hline Erythrocyten: & $\begin{array}{l}\text { Beginn } \\
\text { Ende }\end{array}$ & $"$ & $"$ & $\begin{array}{l}2,95 \\
4,03\end{array}$ & $\begin{array}{l}2,96 \\
4,60\end{array}$ & $\begin{array}{l}2,95 \\
5,47\end{array}$ & $\begin{array}{l}3,13 \\
4,83\end{array}$ \\
\hline Quotient: & $\begin{array}{l}\text { Beginn } \\
\text { Ende }\end{array}$ & $"$ & $"$ & $\begin{array}{l}0,62 \\
0,86\end{array}$ & $\begin{array}{l}0,59 \\
0,83\end{array}$ & $\begin{array}{l}0,62 \\
0,92\end{array}$ & $\begin{array}{l}0,43 \\
0,98\end{array}$ \\
\hline
\end{tabular}

Aus Tabelle V und VJ geht hervor, dass im Durchschnitt bei Behandlung mit Eisen + Arsenik eine rasche Besserung der Chlorose erzielt wird, so dass schon am Ende der 3. Woche, häufiger allerdings am Ende der 4. und 5. Woche Hämoglobinwerth und Erythrocytenzahl die Norm erreichen, oder wenigstens nicht mehr weit davon entfernt sind.

Ars. + Ferr. wurde meist innerlich gegeben ausser in einem Falle, bei dem sich die subcutanen Injectionen nicht als erfolgreicher, sondern eher als etwas langsamer wirkend, im Vergleich mit der inneren Behandlung der übrigen Fälle erwiesen.

Vergleichung der therapeutischen Wirkung ausschliesslicher Eisentherapie mitderWirkung der combinirten Behandlung (Eisen + Arsen.).

Um festzustellen, ob der Heilungsprocess bei der Behandlung der Chlorose mit Eisen allein sich unterscheidet von dem Verlaufe des 
Heilungsprocesses bei der combinirten Behandlung mit Ars. + Eisen vergleichen wir im Folgenden unsere Krankengeschichten in der Weise, dass wir berechnen, wie gross die therapeutische Leistung der verschiedenen Behandlungsmethoden innerhalb einer bestimmten Zeit $(2,3$, 4, 5 Wochen) hinsichtlich Zunahme an Hb. und Er. beträgt.

Ferner lässt sich aus denjenigen Fïllen, bei denen eine genügende Zahl von Beobachtungen vorliegt, berechnen, wie gross die Zunahme an $\mathrm{Hb}$. und Er. in den einzelnen Wochen während der Behandlungsdauer betrïgt, indem wir die Hb.- Werthe und die Zahl der Er. zu Beginn der Behandlung vergleichen mit den entsprechenden Werthen am Ende der 1. Woche der Behandlung und diese Werthe nun wieder vergleichen mit den entsprechenden am linde der 2. Behandlungswoche.

Diese Zusammenstellungen finden sich in den folgenden Tabellen VII und VIII.

Tahelle VII. Vergleichung der therapeutischen Resultate, welche durch die verschiedene Therapie innerhalb gleicher Zeit erzjelt wurden.

A. Hämoglobin.

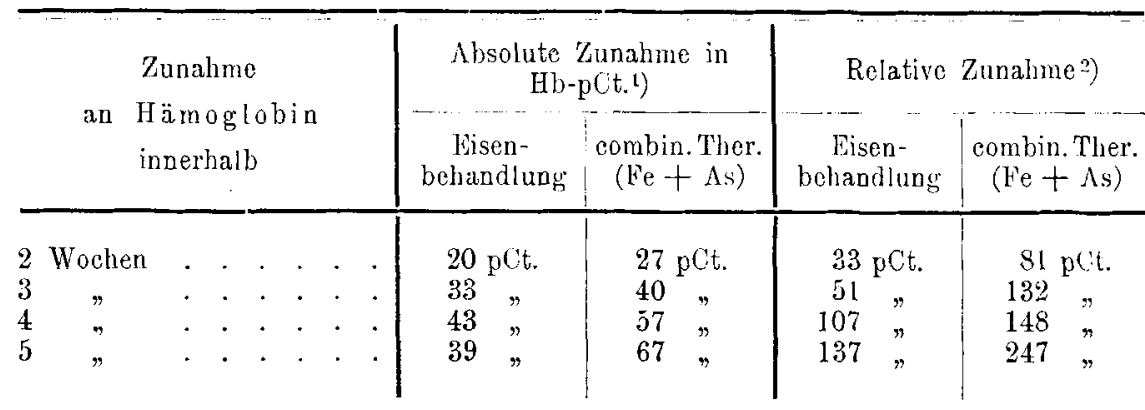

B. Erythrocyten.

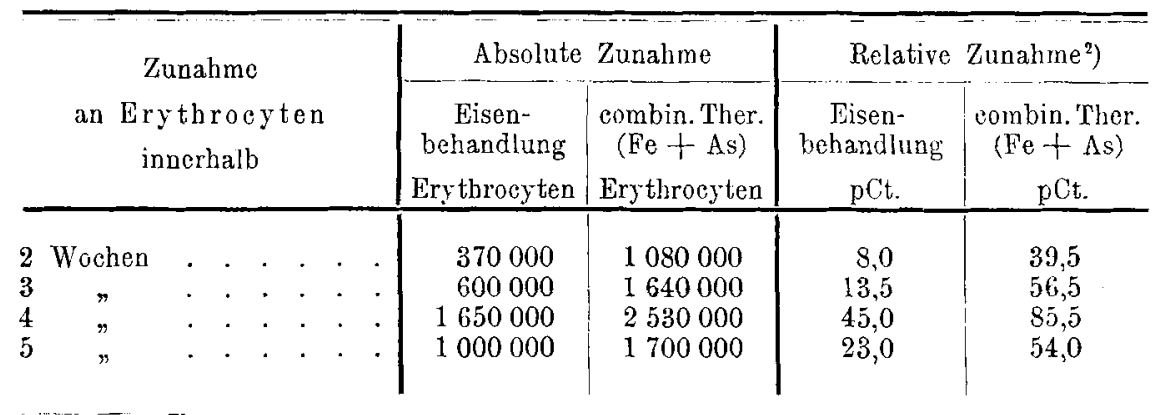

1) Zur Berechnung der absoluten Zunahme an Hämoglobin sind die Werthe umgerechnet auf "corrig. pCt." unter Annahme des $\mathrm{Hb}$-Werthes $70=100 \mathrm{pCt}$. Diese Zahlen repräsentiren somit die absolute Zunahme des Blutes an corrig. Hämoglobinprocenten!

2) Die Berechnung der relativen Zunahme an $\mathrm{Hb}$ und Erythrocyten giebt uns Aufsehluss, um wie viel Procent des Bestandes dieser innerhalb dor betreffenden Vergleichsperiode zugenommen hat. In der vorliegenden Tabelle handelt es sich also bei der "relativen" Zunahme um die Zunahme in Procenten des Initialbestandes. 
Tabelle VIII. Vergleichung der therapeutischen Resultate, welche durch die verschiedene Therapic innerhalb der einzelnen Wochen der Be. handlungszeit erzielt wurden.

\begin{tabular}{|c|c|c|c|c|}
\hline & \multicolumn{2}{|c|}{$\begin{array}{c}\text { Absoluted) Zunahme an } \\
\text { Hämoglobin }\end{array}$} & \multicolumn{2}{|c|}{$\begin{array}{c}\text { Relative1) Zunahme an } \\
\text { Hämoglobin }\end{array}$} \\
\hline & Eisentherapie & $\begin{array}{c}\text { combin. Ther. } \\
\left(\mathrm{Fe}^{\mathrm{e}}+\mathrm{As}\right)\end{array}$ & Eisentherapio & $\begin{array}{c}\text { combinirte } \\
\text { Therapie }\end{array}$ \\
\hline \multirow[t]{2}{*}{ 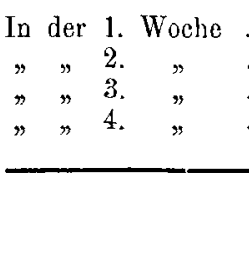 } & $\begin{array}{rl}13,5 & \mathrm{pCt} \\
9,4 & \\
9,6 & " \\
11,0 \quad "\end{array}$ & $\begin{array}{l}13,0 \mathrm{pCt} . \\
17,0 " \\
14,0 " \\
10,6 "\end{array}$ & $\begin{array}{l}21,4 \text { pCt. } \\
13,1 " \\
12,2 " \\
11,7 "\end{array}$ & $\begin{array}{l}23,3 \text { pCt. } \\
34,3 " \\
20,7 " \\
12,0 "\end{array}$ \\
\hline & \multicolumn{2}{|c|}{$\begin{array}{c}\text { Absolute Zunahme an } \\
\text { Erythrocyten }\end{array}$} & \multicolumn{2}{|c|}{$\begin{array}{l}\text { Relative Tunahme an } \\
\text { Erythrocyten }\end{array}$} \\
\hline 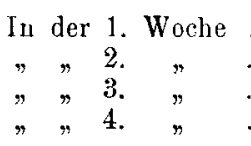 & $\begin{array}{l}610000 \\
170000 \\
270000 \\
300000\end{array}$ & $\begin{array}{l}510000 \\
570000 \\
550000 \\
340000\end{array}$ & $\begin{array}{c}15,4 \mathrm{pCt} . \\
3,7 \% \\
5,8 \% \\
6,5 \%\end{array}$ & $\begin{array}{rl}17,5 & \mathrm{pCt} . \\
16,7 & \Rightarrow \\
13,6 \% \\
7,4 \quad "\end{array}$ \\
\hline
\end{tabular}

Aus diesen Tabellen geht nit Evidenz hervor, dass die combinirte Therapie einen sehr viel günstigeren Einfluss auf die Chlorose ausübt, als die reine Eisentherapie. Der Gesammterfolg einer combinirten Therapie mit Eisen + Arsen ist ein viel besserer als derjenige der Eisenbehandlung allein und zwar sowohl hinsichtlich der Regeneration des Hämoglobins als auch hinsichtlich des Ersatzes der rothen Blutkörperchen.

Die Berechnung der relativen Zunahme giebt uns an, wie viel pCt. des noch vorhandenen $\mathrm{Flb}$. und der Erythrocyten innerhalb der Behandlungszeit gewonnen wurden. Dieser Berechnung ist insofern eine Bedeutung beizumessen, weil es in praxi für die Patienten gewiss von Wichtigkeit ist, wenn die relative Verbesserung seines Blutbefundes eine beträchtliche Grösse erreicht, selbst wonn die absolute Verbesserung nicht eine sehr grosse zu nennen ist. So ist z. B. für eine Patientin mit $20 \mathrm{pCt}$. Hb. eine absolute Zunahme von $5 \mathrm{pCt}$. = relative Zunahme von $25 \mathrm{pCt}$. eine sehr viel wichtigere und prognostisch günstigere Erscheinung als eine analoge Zunahme von 5 pCt. bei einer Patientin mit $40 \mathrm{pCt}$. Hb., wo also die absolute Zunahme dieselbe, die relative Zunahme aber nur $12,5 \mathrm{pCt}$. beträgt. Es hat sich nun gezejgt, dass gerade diese relative Zunahme an Hb. und Er. bei der Behandlung mit Eisen + Arsenik sehr viel grösser ist als bei der reinen Eisen-Behandlung.

Besondere beweisende Bedeutung möchten wir der Tabelle VIII beimessen, welche uns ein Bild giebt über die Fortschritte der Regeneration des Hämoglobins und der Erythrocyten in den einzelnen Wochen der Behandlung. Während in der ersten Woche ein Unterschied des Einflusses von Eisen einerseits und Eisen + Arsenik andererseits nicht in wesentlichem Grade besteht, zeigt sich in der zweiten und dritten Woche eine

1) Ueber die Bedeutung des Ausdruckes „absolute und relative /unahme" vergleiche die Bemerkung zu Tabelle VII. In der vorliegenden Tabelle VIII ist die relative Zunahme in Procenten des Bestandes des Anfangstages der Woche ausgedrückt. 
ganz bedeutende Verschiedenheit des Hejlungsfortschrittes zu gunsten der combinirten Behandlung. Sowohl dic absolute als auch die relative Zunahme an $\mathrm{Hb}$. und auch an Er. erreicht unter der combinirten Behandlung. ganz bedeutend höhere Werthe ais unter der rcinen Eisen-Therapie. In der nun folgenden vierten Woche dagegen sind die Fortschritte wieder sozusagen die nämlichen. Die Ueberlegenheit der combinirten Behandlung macht sich also in erster Jinie in einem ganz bedeutend rascheren Fortschreiten des Heilungsprocesses in der zweiten und dritten Behandlungswoche gegenüber der reinen Ferrumbehandlung geltend.

Es erscheint vielleicht auffällig, dass ein so grosser Unterschied zwischen combinirter Behandlung und reiner Eisenbehandlung nachweisbar ist, da wir doch gefunden hatten, dass Arsenik allein keine wesentliche Beeinflussung der Chlorose zu erzielen vermag. Die Wirkung der combinirten Behandlung ist somit nicht einfach als die Summe der $\Lambda$ rsenikbehandlung + Eisenbehandlung aufzufassen, sondern es müssen hier noch andere Momente die Wirksamkeit beeinflussen. Möglicherweise ist der Vorgang in der Weise zu deuten, dass das Knoehenmark unter Anwesenheit von reichlichen Nengen von Eisen, welches auch schon die Regenerationthätigkeit mächtig anzurcgen im Stande ist, durch Arsenik eine weitere intensive Stimulirung erfährt, vielleicht durch eine Art von Katalyse, welche der Arsenik allein ohne Anwesenheit von reichlich Lisen nicht auszulösen vermag.

Wenn man auf diese hypothetische Deutung verzichtet, so kann man die empirische Thatsache auch unter die in newerer Zeit in immer ausgedehnterem Massstab anerkannten therapeutischen Erfahrungen subsumiren, dass in sehr vielen Fällen die Combination von Arzneimitteln nicht nur stärker sondern auch zuweilen clualitativ anders wirkt, als es der Summe der combinirten Wirkung entspricht. Das heisst - es kann ein Arzneimittel durch Combination mit einem anderen Wirkungen erlangen, welche ihm für sich allein nicht zukommen.

Die Verhältnisse der combinirten Aryneimittelwirkungen sind bekanntlich in letzter Zeit in ausgedehntem Masse systematisch durch Bürgi ${ }^{13}$ ) und seine Schüler ${ }^{14}$ ) tiercxperimentell erforseht worden. Diese Untersuehungen erstreckten sich hauptsäehlich auf die Combination von narkotischen Mitteln und fübrten zu ciner Reihe von interessanten Resultaten, welche es Bürgi erlaubten bestimmte Regeln über die Ar zneicombinationen aufustellen. Bürgi hebt verschiedene Male hervor, dass eine relativ unwirksame Substanz durch die Combination mit einer wirksamen ebenfalls sehr wirksam werden kann. (Beispiele: Brom bei Kaninchen in Verbindung mit anderen Narcotica, Skopolamin in Combination, ete.) In ähnlicher Weise würde somit nach Bürgi auch die ron uns aufgefundene Thatsache $z u$ erklïren sein, dass das an und für sich nach unseren Versuchen bei der Chlorose praktisch vollständig unwirksame oder nur minimal wirksame, aber wio Thierversuche und Lrfahrungen bei der perniciösen Anämic zeigen, doch das Knochenmark beeinflussende Arsenik und das Eisen sich in ihrer Wirkung gegenseitig verstärken.

Die Resultate meiner Arbeit lassen sich zusammenfassen im Folgenden. 


\section{Schlussfolgerungen.}

1. Die Behandlung der Chlorose mit Arsenikpräparaten, speciell Acidum arsenicosum intern oder subcutan, ist allein nicht im Stande, eine wesentliche Verbesserung des Zustandes, Zunahme des Hämoglobins oder Vermehrung der rothen Blutkörperchen, hervorzurufen. Die Wirkungsfähigkeit des Arseniks bei der Chlorose steht im grössten Gegensatz zur Wirkung des Arseniks bei der perniciösen Anämie. Bei letzterer Krankheit ist ja bekanntlich die Arsenikbehandlung häufig von bestem Erfolg. Die vollständig verschiedene Wirksamkeit der Arsenikbehandlung bei diesen beiden Erkrankungen der blutbildenden Organe muss auf der Verschiedenheit des Wesens derselben beruhen.

2. Die Behandlung der Chlorose mit Eisen (Ferrum sulfuricum) in Form von Blaud'schen Pillen bewirkt meistens eine rasche Zunahme des Hämoglobingehaites und bei verminderter Erythrocytenzahl auch eine Vermehrung derselben. Dabei zoigt sich nur ein geringer Unterschied der Regeneration des Hämoglobins, und auch der Erythrocyten in den einzelnen Behandlungswochen.

3. Die Behandlung der Chlorose mit Eisen + Arsenik giebt im Durchschnitt eine wesentlich beschleunigte Besserung des Zastandes im Vergleich zum Heilungsverlauf der Chlorose mit blosser Eisenbehandlung. Man findet die intensivste Zunahme an Hämoglobin schon in der 2. und 3. Woche der Behandlung, und eine 2-3mal intensivere Neubildung von Erythrocyten als bei blosser Eisentherapie.

4. Der Behandlung der Chlorose mit Eisen + Arsenik ist in allen Fällen im Interesse einer möglichst raschen Heilung der Vorzug zu geben. Die Dosis beträgt dabei 3 mal täglich $0,002-0,003 \mathrm{~g}$ Acidum arsenicosum. Diese Dosen, combinirt mit den gewohnten Dosen von Eisen, genügen, eine wesentliche Beschleunigung im Heilungsverlauf der Chlorose gegenüber denjenigen Fällen, welche mit Eisen allein behandelt wurden, zu erzjelen.

5. Die verstärkte therapeutiscbe Wirkung der Eisen-Arseniktherapie, die empirisch bekannt, hier experimentell bestätigt wird, ist keine blosse Additionswirkung von Eisen- und Arsenwirkung, sondern ist in der Weise aufzufassen, dass der Arsenik, obschon für sich allein nicht wirksam, unter gleichzeitigem Einfluss einer an und für sich schon wirksamen Eisenbehandlung die regenerativen Funktionen der blutbildenden Organe der an Chlorose erkrankten Patientinnen in vermehrtem Masse anzuregen vermag.

\section{Literatur,}

1) Jahresbericht über die Leistg. u. Fortschr. in d. ges. Med. Jahr 1903.

2) Lehrbuch d. inn. Med.

3) Lehrbuch d. spec. Pathol. u. Ther. d. inn. Krankh.

4) Jahresbericht über die Leistg. u. Fortschr. in d. ges. Med. Jahr 1900.

5) Med. Klinik. 11. Juli 1909. No. 28.

6) Jahresbericht über die Leistg. u. Fortschr. in d. ges. Med. Jahr 1902. 


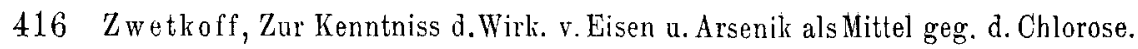

7) Ebenda. Jahr 1900.

8) Lehrbuch d. inn. Med.

9) Lehrbuch der spec. Pathol. u. Ther. d. inn. Krankh.

10) Med. Klinik. 16. Mai 1909. No. 20.

11) Experimentelle Pharmakologie.

12) Virchow's Arch. 1910. Bd. 164.

13) Bürgi, Deutsche med. Wochenschr. 1910. H. 1 u. 2. - Derselbe, Zeitschr. f. Balneol. 3. Jahrg. No. 14. - Derselbe, Schweiz. Rundschau f. Med. 1911. - Derselbe, Zeitschr. f. exper. Path. u. Ther. Bd. 8.

14) Hauckold, Zeitschr. f. exper. Pathol. u. Ther. Bd. 7.

15) Lindemann, Ebenda.

16) Herzenberg, Ebenda. Bd. 8.

17) Zellen, Ebenda.

18) Katzenelson, Ebenda.

19) Saradschian, Ebenda.

20) Lomonos off, Ebenda.

21) Dr. Döbeli, Monatschr. f. Kinderheilkunde. Bd. 9. No. 8.

22) Bergien, Inaugural-Dissertation. Bern 1910.

23) Beinaschewitz, Therap. Monatshefte. Jahrg. XXIV. Okt. 1910. 